\title{
Honorarkampf
}

\section{Kassen sitzen auf ihrem Schatz}

Die Honorarverhandlungen für 2013 sind trotz eines Schiedsspruchs im Erweiterten Bewertungsausschuss gescheitert. Trotz historischer Geldpolster wollen die Krankenkassen den Vertragsärzten lediglich eine MiniErhöhung zugestehen. Der Hausärzteverband ist empört, will aber nicht um jeden Preis "streiken" lassen. Die Gründe stehen in unserer Partner-Zeitschrift "Der Hausarzt".

Es ist ein beispielloser Vorgang. Die Reserven der Gesetzlichen Krankenversicherung (GKV) sind im September auf 21,8 Milliarden Euro angeschwollen. Viele Kassen haben so viel Geld gebunkert, dass sie sogar die zulässige Obergrenze von 1,5 Monatsausgaben überschreiten. Sie wissen im wahrsten Sinne gar nicht mehr, wohin mit dem vielen Geld.

\section{Politik der Nadelstiche eingeleitet}

Auf der anderen Seite steht das offizielles Ergebnis der Honorarverhandlungen für 2013, in denen die Ärzte hatten 3,5 Milliarden Euro gefordert hatten: Der unabhängige Schlichter Jürgen Wasem verfügte ein Plus von 270 Millionen Euro. Der GKV-Spitzenverband hatte sogar ein Minus gewollt.

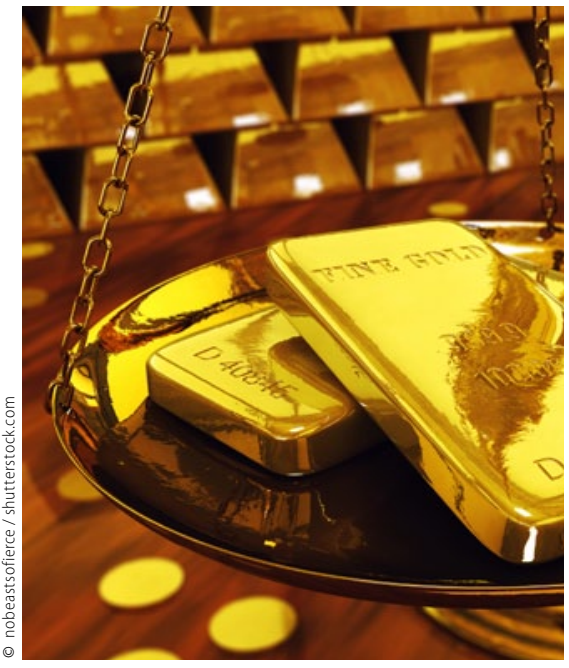

Gold im Überschuss? Ärzte fordern höhere Beiträge.
Wie passt das zusammen? Gar nicht, finden einstimmig alle ärztlichen Berufsorganisationen, von den Verbänden über die KVen bis zu den Kammern. Die Vertragsärzte fühlen sich von den KassenKrösussen vergackeiert - und haben eine „Politik der Nadelstiche“ eingeleitet, wie es von der Kassenärztlichen Bundesvereinigung heißt. Sie ruft die Mediziner zunächst dazu auf, bestimmte bürokratische Dienste zu verweigern, verweist aber auch explizit auf die Urabstimmungen zu Praxisschließungen, die in vielen Verbänden laufen.

\section{Stellenwert der hausärztlichen}

Versorgung darf nicht untergehen

Auch der Hausärzteverband als größte Ärztegemeinschaft in Deutschland vertritt klar die Ansicht, dass die verfügte Honorarsteigerung mit dem realen $\mathrm{Be}$ darf nichts zu tun hat. Für den Bundesvorsitzenden, Ulrich Weigeldt, offenbart die Causa aber auch das Versagen des Kollektivvetragssystems. Die Hausärzte würden die Maßnahmen der KBV unterstützen - wenn diese sich zur Abwechslung auch einmal wahrhaftig für die Hausärzte einsetzt. „Der besondere Stellenwert der hausärztlichen Versorgung kann und darf nicht undifferenziert in der pauschalen Honorardiskussion untergehen", mahnt Weigeldt.

Der Verband hat dafür eine Liste von Forderungen aufgestellt, die die KBV in den Verhandlungen berücksichtigen soll - damit nicht am Ende das Geld bei den Fachärzten landet. Lesen Sie mehr in „Der Hausarzt“!

(Cornelius Heyer) $\frac{\text { Hier steht eine Anzeige }}{\text { This is an advertisement }}$

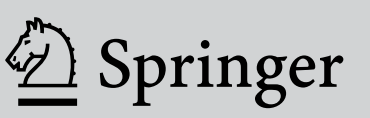

